\title{
A Design Method of Tele-Rehabilitation Platforms for Post-Stroke Patients Based on Consumer Technology
}

\author{
Alberto ARRIGONI ${ }^{\mathrm{a}}$, Michele CUMETTI ${ }^{\mathrm{a}}$, Andrea GRECO ${ }^{\mathrm{a}}$, Luca SOLIVERI ${ }^{\mathrm{a}}$ and \\ Andrea VITALI ${ }^{\mathrm{a}, 1}$ \\ ${ }^{a}$ University of Bergamo, Bergamo, Italy
}

\begin{abstract}
Background: Telerehabilitation represents a new cutting-edge method in the treatment of patients suffering from motor and cognitive disorders caused by stroke. Even if there exist dedicated devices able to track patients' movements to evaluate the performed rehabilitation exercises, they require specific settings necessary for a correct and simple use at the patient's home. If we consider the recent pandemic situation and the lockdown condition, which made difficult the access to these products, post stroke patients may be not able to perform home rehabilitation. Objectives: the goal of this work is the design of a specific method to develop a telerehabilitation platform for post-stroke patients using consumer technologies without involving ad-hoc devices. Method: Open-source tools have been investigated for speeding up the development starting with the medical knowledge. Results: a group of four healthcare technologies engineering students with no specific skills about computer science has developed a platform in four months using the design method. Conclusion: the presented method allowed the development of a clinical knowledgebased web platform for post-stroke patients totally based on consumer technology.
\end{abstract}

Keywords. Telerehabilitation, Stroke rehabilitation, Virtual Reality Exposure Therapy, Rehabilitation Research

\section{Introduction}

A systematic analysis for the Global Burden Disease Study showed that in 2016 there were 116.4 million DALYs due to stroke and 80.1 million prevalent cases of stroke globally including 5.5 million deaths [11]. Upper extremity function and cognitive impairments are common outcomes following stroke events, causing affected people a worsening of their lifestyle and a loss in work opportunities. Despite post-stroke rehabilitation therapy being generally provided in clinical facilities, many patients do not draw the maximal benefit from it. The major problems that come across during the traditional rehabilitation path are related to access issues, costs and scarce adherence.

Tele-rehabilitation-based programs may throw down the cited barriers. This form of telemedicine allows delivering remote rehabilitation services directly to the patient's home. Technology can overcome access obstacles to quality care, including the burden to travel for in-patient therapy, along with the consequent costs, scheduling demand issues and shortage of trained therapy providers. Moreover, tele-rehabilitation systems

\footnotetext{
${ }^{1}$ Corresponding Author: Andrea Vitali, University of Bergamo, Italy, E-Mail: andrea.vitali1@unibg.it
} 
have proven to enhance post-stroke rehabilitation's outcomes by promoting adherence to rehabilitation therapies, boosting the brain plasticity [6]. Besides, a remotely provided rehabilitation service may positively affect the patient selection in terms of clinics, enlarging the choice opportunities by making available care providers which are located far from home. Another technological solution, that fits well along with telerehabilitation and may increase patient engagement during therapies, is the deployment of serious games. These are games with non-recreational intent but designed and developed pursuing a specific goal, typically linked to educational or training purposes under the scope of many contexts. These include the rehabilitation background. The key concept behind this kind of game is to provide a valuable, fun and engaging rehabilitation path. Despite all the listed advantages, virtual-aided rehabilitation cannot be considered the first therapy choice yet due to the still scarce availability of low-cost technologies and the low confidence of the target population, largely represented by elders, when it comes to manage technological solutions. Although the Human-Computer Interaction (HCI) research advances in interactive computer systems, usability barriers still obstruct access to this kind of solutions, discouraging its diffusion among users [15].

The coexistence of these pros and cons makes it interesting to design a methodological approach for the development of tele-rehabilitation platforms for taking advantage of the benefits related to the remote modality while avoiding the drawbacks. This point means achieving a system able to provide patients with efficient and stimulating treatments, whose progress can be monitored remotely by clinicians. The goal is the investigation of methods and tools to develop a platform patient and medical centered by exploiting low-cost and user-friendly technologies.

\section{Scientific Background}

While tele-rehabilitation proved to be a relevant resource in the clinical field, several research projects started in this regard. As shown in the study of Dodakian et al. [4], a platform for remote rehabilitation can provide patients a wide range of features besides the rehabilitative service, specifically sections dedicated to clinical education and doctorpatient interaction.

Regarding the design of serious games, to increase engagement can be useful the compliance with some key principles already examined by companies of the videogame market. These concepts transposed to the rehabilitation field represent a valuable tool to make the treatment successful in avoiding scarce adherence. The mentioned principles, indicated by Barret et al. [1] and Burke et al. [3], are the following: (i) a serious game must be meaningful to obtain clinical evidence on the patient condition from each exercise; (ii) the rehabilitation exercises need to be customizable in order to match the ability of each patient and thus, the level of difficulty must be personalized according to the rehabilitation progress; (iii) the game must be able to show the improvements with quantifiable feedback to make patients realize the value of their efforts.

To achieve medical relevance, the games must examine parameters useful to understand the rehabilitation progress during the time permitting to adjust the program as required by the specific case. Examples of these factors can be time, score, gameplay speed, the eventual number of obstacles avoided successfully in a platform game and the trajectories followed by the limb during the exercise. The challenging aspect must be correctly balanced to determine the success of the therapy: an exercise with a biased level of complexity can lead to discontent both due to an excessive simplicity or difficulty. 
Boredom or frustration are harmful consequences to the observance of the training, which is not something trivial. As pointed out by Goršič et al. [8], people with chronic cognitive or arm impairment should exercise intensely to regain their abilities, but frequently a lack of motivation leads to poor rehabilitation outcome. This issue does not concern only the traditional rehabilitation approach as a recent study showed that, even if a therapist prescribes a technology-supported exercise, only about $30 \%$ of unsupervised patients will comply with the rehabilitation regimen [7]. An additional promising way to increase motivation may be to include a social component within the rehabilitation games, allowing patients to play together with other people. This implementation could have a positive impact in the acceptance and enjoyment of the therapy, leading to long term achievements. Tele-rehabilitation solutions may vary greatly from employing commercial gaming consoles to rehabilitation-specific products up to expensive Virtual reality customized programs. The survey study of Hung et al. [10] underscores a positive trend of game-based rehabilitation systems using consoles such as tablets, Kinects, and Wii remotes. The same trend was detected by Laver et al. [13] in their 2017 update of a Cochrane Review about the virtual reality in stroke rehabilitation, where the number of studies using commercially available gaming consoles increased from 6 to 22 since 2015 .

On some occasions, also the webcam device embedded in each PCs has been exploited, and this is the case of Burke et al. [2], who developed a series of games based on color tracking algorithms working on webcam footages. The spread of smartphones within everyone's reach is also something to consider for preparing solutions that take advantage of mobile apps. Lee Hughes and colleagues [9] evaluated which features are worth to be included in a mobile product designed as a platform for tele-rehabilitation. According to them, this app should include a section dedicated to the patient and one to the doctor. The latter must be designed to include the privilege of modifying the treatment plan available on the patient's interface, as well as the possibility to check the training performance and the medical information which need to be kept into account, like medications and test results. A section for doctor-patient communication is also functional for efficient therapy, such as a calendar that displays the periodic appointments with the care provider team.

Considering the characteristics of the context and the scientific background, the aim of this work has been the definition of a specific method for developing an innovative multi-featured platform for cognitive and motor telerehabilitation intended for poststroke patients.

\section{Method and tools}

Two target features have driven the requirement analysis to make the method pioneering in exploring alternative approaches than the current available solutions, also providing new advantages [12], i.e.:

- the use of advanced tools for facilitating and speeding up the development,

- the exclusive deployment of consumer technologies which are usually present in people's homes, without involving any investment or hardship related to the supply of the necessary equipment (e.g., the use of a Microsoft Kinect device for human motion detection). This intention arose from a nowadays striking need if considering the recent pandemic situation and the lockdown condition 
which has highlighted sustainability limits by health systems in managing all the care processes without more agile solutions. This intention led the research to the employment of an artificial neural network model for achieving the motion tracking required by the rehabilitation aim, which is an area that needs further continuous explorations, despite being a recognized valuable tool for general tracking in computer vision [14].

Specifically, the realized method considers two mobile applications, addressing the doctor and the patient, one game for cognitive rehabilitation and one for the upper limb motor rehabilitation based on a hand-tracking interaction style. All the modules communicate through internet connection with a server database. By starting from the target features previously described, the first step has concerned the analysis and selection of technological tools required for respecting all the features required for the realization of the technological platform.

The chosen game editor to develop serious games has been the free Unity 3D because of: (i) no need of high skills in computer graphic development; (ii) multiplatform; (iii) useful add-ons for interfacing hand-tracking devices.

To obtain a deep learning model for the hand-tracking system, the selected tool has been the Python programming language with PyCharm as the development environment.

Android Studio has been chosen for building the mobile applications. The communication among all the applications has been implemented with MySQL DBMS and LoopBack [16]. The former allows the design of the database; the latter provides automatic REST API generation for the server.

According to the selected technological tools, a basic software architecture has been designed and depicted in Figure 1. The whole web platform is based on the standard HTTP protocol for the data exchanging.

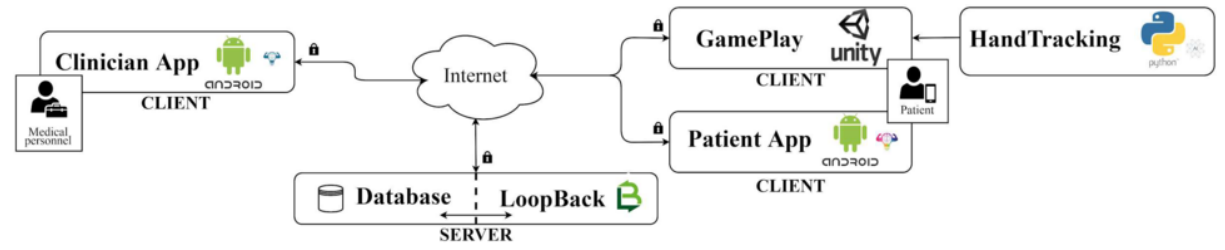

Figure 1. Basic software architecture of a tele-rehabilitation platform for post-stroke patients.

\section{Discussion}

By starting from the proposed method, a platform has been realized to investigate the potentialities of the proposed approach.

\subsection{Development of serious games}

The developed serious games are characterized by different functionalities such as different game modalities, distinct levels of difficulties, time and error-based scores, besides a simple interface featured by sounds and instructions. Two different types of games have been designed based on functional and motivational parameters: 
- Cognitive rehabilitation: the game is focused on stimulating the patient's memory, inducing him to remember the exact sequence of some cooking recipes (Figure 2a). Exploiting the mouse interface interaction, is asked to the patient to remember the correct sequence of ingredients to make a specific dish, projecting the patient in a familiar home-looking virtual environment.

- Motor-skills rehabilitation: stimulating the patient to recover arms' functionalities. The webcam's footage processing algorithm automatically recognizes the hand of the patient and tracks its coordinates to lead the game's character in avoiding obstacles within a funny and stimulating virtual environment designed to enhance the adherence to the therapy (Figure 2b).

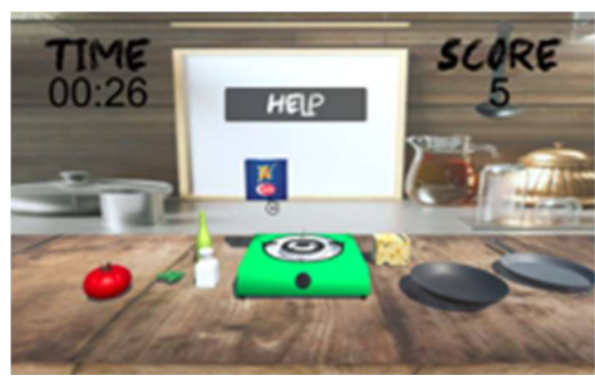

(a)

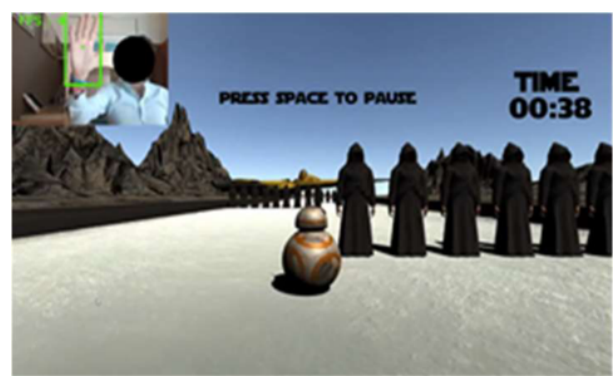

(b)

Figure 2. Unity 3D serious games: Game's environment for cognitive rehabilitation (a); Gameplay and tracking system which determines the movement of the player for motor-rehabilitation (b).

\subsection{Hand tracking by means of artificial intelligence}

In order to avoid the use of specific hand-tracking devices, the motor rehabilitation game's design concerns the employment of an AI computer vision algorithm. Specifically, it consists of an open-source convolutional neural network based on the SSD MobileNet architecture and, according to the author, trained in cloud on a GPU machine using the Tensorflow API and employing the Egohands Dataset [5], reaching a mean Average Precision (mAP) value of 0.9686 and a total loss of 2.575 [18]. The Python scripts were appropriately modified to fit the needs of the intended application, both in terms of parameters optimization, with the number of target hands set to 1, and the assessment of a balanced certainty threshold, as well as new features implementation. Precisely, the algorithm's outcome was configured to provide, in addition to the bounding boxes drawing, also the visual information of each box centre and the tracking record of its coordinates in order to post them to the game application in real-time. About tracking this point, an interpolation was implemented for shifting the horizontal range of movement from zero up to the frame width value, to a reduced symmetrical interval that allows the movement of the game's character on both the left and right directions with respect to the centerline of the frame.

The obtained motion coordinates were sent to the rehabilitation game through web sockets based on UDP (i.e., the standard User Datagram Protocol). The optimized and adapted algorithm was then converted in a Windows executable application to make it easily accessible without installing any resources or possessing any skills. The next step was to integrate the hand tracking data to control a character moving continuously forward on a path with obstacles. The idea pursued was to convert the horizontal 
coordinates information in a lateral thrust force operating on the player, which behaves like a real rigid body. This feature provided a further measure to raise the challenge of the game in terms of entertainment and competition by involving the practice of a motorcognitive skill helpful for rehabilitation. A Unity3D script has been developed for using the UDP Socket communication to receive the position data to be exploited by the character's movement scripts in generating the motion force during the gameplay. The tracked movement is automatically saved on the MySQL database using the REST API designed with Loopback.

\subsection{Development of the mobile applications}

The mobile application for patients contains different features to carry out the telerehabilitation in an engaging environment: background music, button's sound and an immediate user interface. The app contains a Memory cards game developed for cognitive rehabilitation where the player, turning two cards at a time, must find the pairs with the same front image. A section with motor-rehabilitation training exercises (Figure 3a) for the improvement of daily life tasks is also available. The patient's app also offers a multi-choice quiz with a stroke-educational goal. Finally, a personal agenda shows the dates of medical examinations and the personalized rehabilitation program.

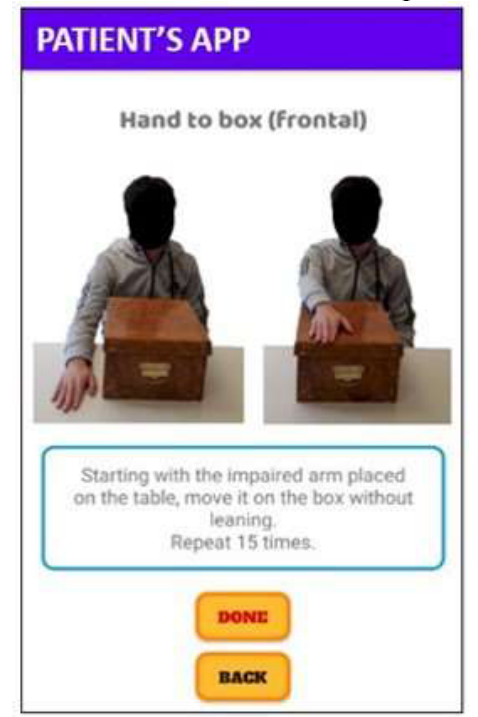

(a)

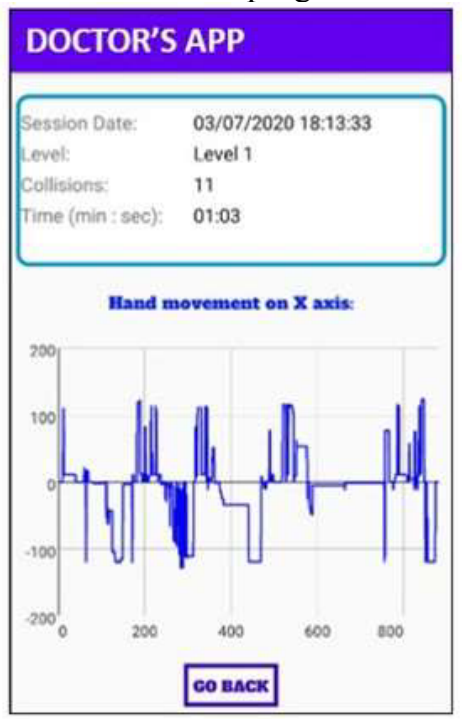

(b)

Figure 3. Mobile applications' features: Training motor-rehabilitation based on the Wolf Motor Function Test (a); rehabilitation results section on the doctor's application (b).

The doctors' mobile app allows the patients' data evaluation. The physician accesses a selection list of activities that can be associated to each specific patient. The application offers a section containing the progress of the motor and cognitive rehabilitation path and the graphical representation of the coordinates, showing the movement of the patient's hand, detected by the hand tracking algorithm (Figure 3b). The doctor visualizes the patient's data and his/her improvements useful to modify the rehabilitation program. Both the mobile apps allow patients and doctors to contact each other by e-mails. All data and information are saved or modified on the MySQL database using the REST API designed with Loopback. 


\section{Discussion}

The presented method has been designed to develop a platform for post stroke telerehabilitation by four students attending a master's degree course focused on medical technologies and their management. The limit of time imposed by the project deadline, as well as the complexity of developing such a product starting from a limited coding experience, were successfully addressed by the designed method and by using the proposed development tools. An initially planned approach was based on the use of a Leap Motion device [17] for realizing the motion tracking required by the motorrehabilitation game but considering the complications that arose with the COVID-19 pandemic outbreak and the resulting impossibility to get the needed equipment during the lockdown, an innovative way has been pursued based on the use of artificial intelligence as suggested by the design method presented. The hand-tracking interaction style has been developed by using a convolutional neural network model working on a video stream. The whole platform for the rehabilitation of post-stroke patients has been developed in approximately 4 months.

The simple hand tracking algorithm implemented has limits compared to other technologies that are very precise and allow to obtain several data, however, there already exist open-source models much more sophisticated than the adopted one which enables to obtain further and useful information on the movement performance, such as pose estimators. The whole platform is based only on consumer technology: the patient can use her/his laptop to play the serious games by means of the traditional webcam to perform the hand-tracking. Therefore, no installation of specific devices is needed at the patient's home.

Specific improvements have been planned to add the cybersecurity requirement (i.e., HTTPS protocol) for data assurance and start a clinical assessment of the telerehabilitation platform by involving post-stroke patients under the supervision of medical experts. At present, the project has not yet been able to collect the opinion of any care provider institutions nor find their involvement due to the particular situation, which has shifted the focus to the emergency management.

\section{Conclusions}

The implemented method constitutes an innovative proposal for the design of a multifunctional system aimed at the telerehabilitation of post-stroke patients. The proposed approach shows how the choice of specific open-source development tools can facilitate the rapid and successful realization of these products. Regarding the utilization of serious games in motor rehabilitation, the proposed method explores a promising new strategy to track upper limbs movement given the advantage of not requiring any additional equipment other than laptops, smartphones and tablets with an embedded webcam. Furthermore, the method considers the continuous advancement of artificial intelligence models whose real-time deployment is becoming more and more accessible thanks to the improvement of the IT computing components. Moreover, the promising value of the suggested approach is clear considering the current spreading of cloud-based gaming systems which would enable in the future the easy access to powerful hardware and therefore the implementation of even more sophisticated tracking algorithms. 


\section{References}

[1] N. Barrett, I. Swain, C. Gatzidis, and C. Mecheraoui. The use and effect of video game design theory in the creation of game-based systems for upper limb stroke rehabilitation. Journal of Rehabilitation and Assistive Technologies Engineering, 3: 2055668316643644, 2016.

[2] J. W. Burke, M. McNeill, D. Charles, P. Morrow, J. Crosbie, and S. McDonough. Serious games for upper limb rehabilitation following stroke. In 2009 Conference in Games and Virtual Worlds for Serious Applications, pages 103-110. IEEE, 2009.

[3] J. W. Burke, M. McNeill, D. K. Charles, P. J. Morrow, J. H. Crosbie, and S. M. McDonough. Optimising engagement for stroke rehabilitation using serious games. The Visual Computer, 25(12):1085-1099, 2009.

[4] L. Dodakian, A. L. McKenzie, V. Le, J. See, K. Pearson-Fuhrhop, E. Burke Quinlan, R. J. Zhou, R. Augsberger, X. A. Tran, N. Friedman, et al. A home-based telerehabilitation program for patients with stroke. Neurorehabilitation and neural repair, 31(10-11):923-933, 2017.

[5] Egohands Dataset. URL http://vision.soic.indiana.edu/projects/egohands

[6] L. V. Gauthier, C. Kane, A. Borstad, N. Strahl, G. Uswatte, E. Taub, D. Morris, A. Hall, M. Arakelian, and V. Mark. Video game rehabilitation for outpatient stroke (vigorous): protocol for a multi-center comparative effectiveness trial of in-home gamified constraint-induced movement therapy for rehabilitation of chronic upper extremity hemiparesis. BMC neurology, 17(1):1-18, 2017.

[7] M. Goršič, I. Cikajlo, N. Goljar, and D. Novak. A multisession evaluation of an adaptive competitive arm rehabilitation game. Journal of neuroengineering and rehabilitation, 14(1):1-15, 2017.

[8] M. Goršič, I. Cikajlo, and D. Novak. Competitive and cooperative arm rehabilitation games played by a patient and unimpaired person: effects on motivation and exercise intensity. Journal of neuroengineering and rehabilitation, 14(1):1-18, 2017.

[9] C. M. L. Hughes, A. Padilla, A. Hintze, T. M. Raymundo, M. Sera, S. Weidner, J. Ontiveros, T. Peng, A. Encarcion, Z. A. Cruz, et al. Developing an mhealth app for post-stroke upper limb rehabilitation: Feedback from us and ethiopian rehabilitation clinicians. Health informatics journal, 26(2):1104-1117, 2020.

[10] Y.-X. Hung, P.-C. Huang, K.-T. Chen, and W.-C. Chu. What do stroke patients look for in game-based rehabilitation: a survey study. Medicine, 95(11), 2016.

[11] C. O. Johnson, M. Nguyen, G. A. Roth, E. Nichols, T. Alam, D. Abate, F. Abd-Allah, A. Abdelalim, H. N. Abraha, N. M. Abu-Rmeileh, et al. Global, regional, and national burden of stroke, 1990-2016: a systematic analysis for the global burden of disease study 2016. The Lancet Neurology, 18(5):439-458, 2019.

[12] V. Kumar. 101 design methods: A structured approach for driving innovation in your organization. John Wiley \& Sons, 2012.

[13] K. E. Laver, B. Lange, S. George, J. E. Deutsch, G. Saposnik, and M. Crotty. Virtual reality for stroke rehabilitation. Cochrane database of systematic reviews, (11), 2017.

[14] P. Li, D. Wang, L. Wang, and H. Lu. Deep visual tracking: Review and experimental comparison. Pattern Recognition, 76: 323-338, 2018.

[15] H. Rex Hartson. Human-computer interaction: Interdisciplinary roots and trends. Journal of Systems and Software, 43(2): 103-118, 1998.

[16] StrongLoop. Loopback 4. URL https://loopback.io/.

[17] Ultraleap. Leap motion device. URL https://www.ultraleap.com/.

[18] D. Victor. Handtrack: A library for prototyping real-time hand tracking interfaces using convolutional neural networks. GitHub repository, 2017. 